\title{
Network approaches to climate science
}

\author{
JingFang Fan $^{1 *}$, Jun Meng ${ }^{1,2}$, XiaoSong Chen ${ }^{3,4^{*}}$, Yosef Ashkenazy ${ }^{2}$, and Shlomo Havlin ${ }^{1}$ \\ ${ }^{1}$ Department of Physics, Bar-Ilan University, Ramat-Gan 52900, Israel; \\ ${ }^{2}$ Solar Energy and Environmental Physics, Blaustein Institutes for Desert Research, Ben-Gurion University of the Negev, \\ Beersheba 84996, Israel; \\ ${ }^{3}$ CAS Key Laboratory of Theoretical Physics, Institute of Theoretical Physics, Chinese Academy of Sciences, Beijing 100190, China; \\ ${ }^{4}$ School of Physical Sciences, University of Chinese Academy of Sciences, Beijing 100049, China
}

Received September 8, 2016; accepted September 22, 2016; published online October 24, 2016

$\begin{array}{ll}\text { Citation: } & \text { J. F. Fan, J. Meng, X. S. Chen, Y. Ashkenazy, and S. Havlin, Network approaches to climate science, Sci. China-Phys. Mech. Astron. 60, 010531 } \\ \text { (2017), doi: } 10.1007 / \mathrm{s} 11433-016-0362-2\end{array}$

Climate and physics are closely related. The governing equations for both the atmosphere and ocean are the Navier-Stokes equations, which describe and quantify the physics of fluids. However, the atmosphere and ocean are very complex due to the interaction of many processes, not necessarily physi$\mathrm{cal}$, and due to the need to model processes that are smaller than the grid resolution. In spite of this complexity, physicists have successfully addressed a wide swath of it using advanced statistical physics methods and techniques. Climate science has been benefited from the physics discipline and vice versa where the "chaos" theory is an excellent example for the interdisciplinary approach as it was basically discovered by Lorenz [1] through his simple model of the atmosphere.

Complex networks approach has emerged as an important tool in the analysis of complex systems in the last two decades and has been applied to a wide variety of disciplines in natural and social sciences [2-5]. A network is composed of nodes and links. For example, airports can represent the nodes in a network and the flights between different airports can be regarded as the links of the network. Another example is the internet. The simple network representation allowed mathematicians and physicists to find interesting phenomena regarding the distribution of node and links and the resilience of

*Corresponding authors (JingFang Fan, email: j.fang.fan@gmail.com; XiaoSong Chen, email: chenxs@itp.ac.cn) the network to "attacks" [6,7]. In recent years, complex networks have been used to analyze, quantify and predict climate phenomena [8-10]; this new developed network was termed "climate networks".

In climate networks the geographical locations (or grid points) are regarded as the nodes in the networks and the level of similarity (correlation) between the records in grid points represents the link of the network and its strength. Various climate data records such as temperature, pressure, winds and precipitation can be used to construct the network. The climate networks approach allows to study the interrelationship between the different locations on the globe and thus represent the global behavior of the climate system and how the energy is transferred from one location to another (teleconnections) [11].

El-Niño is a coupled ocean-atmosphere phenomenon that leads to warming of the eastern tropical Pacific region. It has global impacts of, e.g., enhanced rain in some regions and droughts in other regions, decline in fishery, famine, plagues, political and social unrest, and economic changes. Presently, we have just undergone one of the strongest El-Niño events since 1948 [12,13]. The so-called "Godzilla" El-Niño brought droughts along the U.S West coast [13], and heavy rain to Southern China (CCTV news, http://www.cctv-america. com/2016/05/16/el-nino-brings-heavy-rain-to-southernchina). In fact, this strong event was predicted one year ahead using climate network-This prediction is specially 


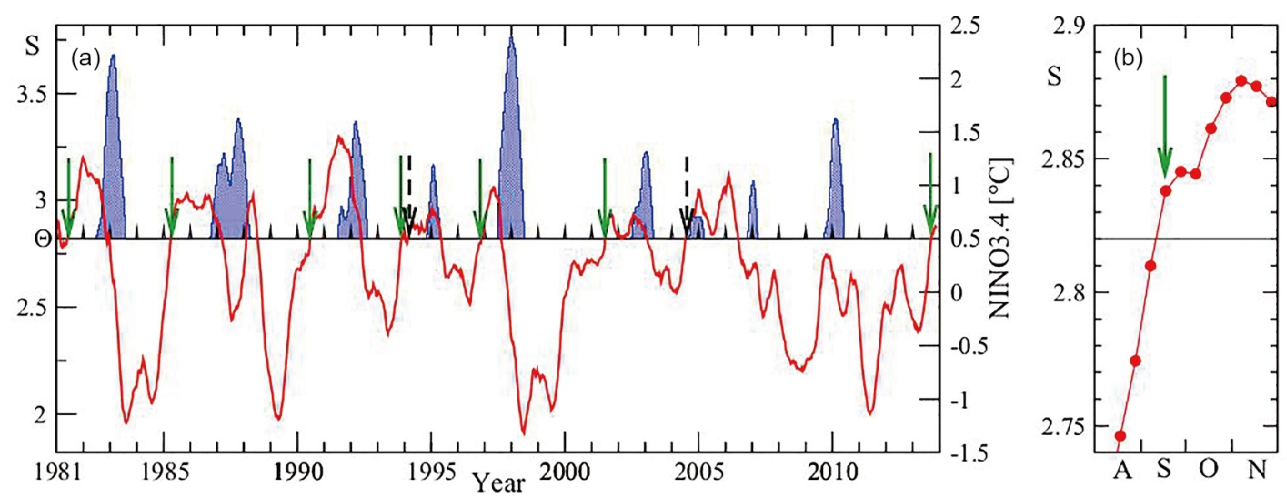

Figure 1 (Color online) The forecasting scheme for El-Niño events (Adapted from Figure 2 of ref. [10]). The forecasting scheme, (a) we compare the average link strength $S(t)$ in the climate network (red curve) with a decision threshold $\Theta$ (horizontal line, here $\Theta=2.82$; left scale) and the standard NINO3.4 index (right scale) between January 1981 and November 2013. When the link strength crosses the threshold from below, outside an El-Niño episode, we give an alarm and predict that an El-Niño episode will start in the following calendar year. The El-Niño episodes (when the NINO3.4 index is above $0.5^{\circ} \mathrm{C}$ for at least $5 \mathrm{mo}$ ) are shown by the solid blue areas. Correct predictions are marked by green arrows and false alarms by dashed arrows; (b) magnification of (a) for August (A), September (S), October (O), and November (N) 2013. The figure shows that by September 17 (green arrow), the optimal decision thresholds have been crossed, forecasting an El Nino event in 2014. In (a), the learning phase (1950-1980) where the optimal thresholds have been learned has been omitted.

interesting as almost all other models failed to predict this strong event so early (Sept. 2013) before the event. See Figure 1, for prediction of El-Niño events using network theory. Many other interesting results regarding El-Niño have been obtained based on the climate network approach [8,10,14-17].

Other climate phenomena such as the North Atlantic Oscillation [18], Rossby waves [19], teleconnection [11], and monsoon [20-22] have been also studied and currently being studied using climate networks.

Climate science is an interdisciplinary science that offers many exciting opportunities and challenges for physicists. Until now, there has been advance in the understanding of the structure and pattern of climate phenomena using network approaches. However, there are many more climate issues that network approaches could be found useful. For example, the prediction of impacts around globe and strength for El-Niño events; the understanding of monsoon; the prediction of earthquakes and the cognition of air pollution. We believe that new ideas and innovations can emerge from the collaboration between physicists and climate scientists, and in this way to improve our understanding regarding the climate system.

This work was supported by the MULTIPLEX EU Project (Grant No. 317532), the Israel Science Foundation, ONR and DTRA, the National Natural Science Foundation of China (Grant No. 1121403). J. F. Fan thanks the fellowship program funded by the Planning and Budgeting Committee of the Council for Higher Education of Israel.

1 E. N. Lorenz, J. Atmos. Sci. 20, 130 (1963).

2 D. J. Watts, and S. H. Strogatz, Nature 393, 440 (1998).
3 A. Barabási, and R. Albert, Science 286, 509 (1999).

4 R. Cohen, and S. Havlin, Complex Networks: Structure, Robustness and Function (Cambridge University Press, Cambridge, 2010).

5 M. Newman, Networks: An Introduction (Oxford University Press, Oxford, 2010).

6 R. Albert, H. Jeong, and A. L. Barabási, Nature 406, 378 (2000).

7 R. Cohen, K. Erez, D. Ben-Avraham, and S. Havlin, Phys. Rev. Lett. 85, 4626 (2000); ibid. 86, 3682 (2001).

8 K. Yamasaki, A. Gozolchiani, and S. Havlin, Phys. Rev. Lett. 100, 228501 (2008).

9 J. F. Donges, Y. Zou, N. Marwan, and J. Kurths, Eur. Phys. J. Spec. Top. 174, 157 (2009)

10 J. Ludescher, A. Gozolchiani, M. I. Bogachev, A. Bunde, S. Havlin, and H. J. Schellnhuber, Proc. Natl. Acad. Sci. 110, 11742 (2013), arXiv: 1304.8039; ibid. 111, 2064 (2014).

11 D. Zhou, A. Gozolchiani, Y. Ashkenazy, and S. Havlin, Phys. Rev. Lett. 115, 268501 (2015).

12 A. F. Z. Levine, and M. J. McPhaden, Geophys. Res. Lett. 43, 6503 (2016).

13 E. Kintisch, Science 352, 1501 (2016).

14 A. A. Tsonis, and K. L. Swanson, Phys. Rev. Lett. 100, 228502 (2008).

15 A. Gozolchiani, K. Yamasaki, O. Gazit, and S. Havlin, Europhys. Lett. 83, 28005 (2008).

16 A. Gozolchiani, S. Havlin, and K. Yamasaki, Phys. Rev. Lett. 107, 148501 (2011), arXiv: 1010.2605.

17 J. F. Fan, J. Meng, Y. Ashkenazy, and S. Havlin, arXiv: 1609.00547.

18 O. Guez, A. Gozolchiani, Y. Berezin, S. Brenner, and S. Havlin, EPL 98, 38006 (2012), arXiv: 1109.3633.

19 Y. Wang, A. Gozolchiani, Y. Ashkenazy, Y. Berezin, O. Guez, and S. Havlin, Phys. Rev. Lett. 111, 138501 (2013), arXiv: 1304.0946.

20 N. Malik, B. Bookhagen, N. Marwan, and J. Kurths, Clim. Dyn. 39, 971 (2012).

21 N. Boers, B. Bookhagen, N. Marwan, J. Kurths, and J. Marengo, Geophys. Res. Lett. 40, 4386 (2013).

22 N. Boers, B. Bookhagen, H. M. J. Barbosa, N. Marwan, J. Kurths, and J. A. Marengo, Nat. Commun. 5, 5199 (2014). 